\title{
Dirac oscillator and nonrelativistic Snyder-de Sitter algebra
}

\author{
M.M. Stetsko* \\ Department of Theoretical Physics, Ivan Franko National University of Lviv, \\ 12 Drahomanov Str., Lviv, UA-79005, Ukraine
}

October 17, 2018

\begin{abstract}
Three dimensional Dirac oscillator was considered in deformed space obeyed to deformed commutation relations known as Snyder-de Sitter algebra. Snyder-de Sitter commutation relations gives rise to appearance minimal uncertainty in position as well as in momentum. To derive energy spectrum and wavefunctions of the Dirac oscillator supersymmetric quantum mechanics and shape invariance technique was applied.
\end{abstract}

\section{Introduction}

The Dirac oscillator represents an example of relativistic exactly solvable quantum model. It was firstly proposed by Itô and collaborators to replace the momentum operator $\mathbf{P}$ in the free particle's Dirac equation by combination $\mathbf{P}-i m \omega \mathbf{X} \beta$ where $\mathbf{X}$ was the position operator, $m$ being the particle's mass and $\omega$ the oscillator frequency. Then unusual accidental degeneracy of the Dirac oscillator's spectrum was investigated by Cook [2]. Supersymmetric approach to the Dirac oscillator was investigated in $[3,4]$. We note that the name Dirac oscillator for this relativistic problem was given by Moshinsky and Szczepaniak [5] who rederived it and shown that in nonrelativistic limit the relativistic hamiltonian becomes a harmonic oscillator with a strong spin-orbit coupling term. The last work renewed interest to the Dirac oscillator and it was examined form different viewpoints, such as covariance properties [6], complete energy spectrum and wavefunctions [7], Lie algebra symmetry [8], shift operators [9], hidden supersymmetry [7, 10, 11, 12], conformal invariance [13], completeness of wavefunctions [14], approach based on Clifford algebra [15]. Some generalization of Dirac oscillator was also considered [16].

The Dirac oscillator model was applied to problems of nuclear and high energy physics. Relativistic many body systems with interactions modelled by the Dirac oscillator hamiltonians with applications to mesons and baryons was considered [17]. Thermodynamics of Dirac oscillators in $1+1$ spacetime was noted to be important in studies of quark-gluon plasma [18]. It was also utilized for developing of effective approach for description of intermediate and short-range components of nucleon-nucleon interaction [19]. Dirac oscillator was used for modelling photon-nucleus scattering [20]. Another area where the Dirac oscillator model was extensively applied is quantum optics. Relation between the Dirac oscillator and relativistic Jaynes-Cummings model was investigated [21]. Mapping of the Dirac oscillator onto Jaynes-Cummings model in case of different dimensions was examined in [22]. In regard to the Jaynes-Cummings model chirality quantum phase transition in $2+1$ dimensional Dirac oscillator subjected to constant magnetic field was investigated [23]. Zitterbewegung behaviour of the Dirac oscillator and possible realization of such a system was considered in $[24,25,26]$. Several attempts to get experimental realization of such a model were made $[27,28]$.

Here we consider the Dirac oscillator from a bit different point of view, namely we solve the Dirac oscillator eigenavalue problem in space with deformed Heisenberg algebra that lead to appearance of minimal uncertainties in position and momentum. The interest to the theories with deformed

*E-mail: mstetsko@gmail.com, mykola@ktf.franko.lviv.ua 
Heisenberg algebra was inspired by investigations in string theory and independently by several approaches to quantum gravity $[29,30,31]$ where it was suggested the existence of a finite lower bound for resolution of length $\Delta X$, so called minimal length. Deformed commutation relations that leads to existence of minimal uncertainty in position and momentum was proposed firstly by Kempf and collaborators [32] and then were investigated from different viewpoints. We point out that only a few quantum mechanical problems are solved exactly, that is to say the harmonic oscillator in one [32] and $D$ dimensions [33], the one- [34] and three-dimensional [35] Dirac oscillator and one dimensional Coulomb-like problem [36]. Lorentz-covariant deformed algebra with minimal length was proposed and 1+1 dimensional Dirac oscillator problem was solved [37]. Minimal uncertainty for momentum can be treated as a consequence of gravity induced decoherence [38]. Uncertainty relation that gives rise to appearance of minimal momentum is also possible in theories with position dependent effective mass [39]. We note that deformed commutation relations with minimal length and momentum were proposed even earlier in context of quantum group theory [40]. Later it was shown that similar uncertainty principle with minimal length and momentum can be obtained in a gedanken experiment of measuring of position in de Sitter space [41]. Deformed algebra with minimal length and momentum was also obtained in context of Triply Special Relativity [42]. It should be noted that basic principles of triply special relativity adopt three fundamental constants and one of them can be identified with a cosmological constant of de Sitter space. In case of deformed algebra with minimal length and momentum only the harmonic oscillator was examined $[43,44,45]$.

Our paper is organized as follows. In the second section an uncertainty relation obtained from deformed algebra is analyzed then the Dirac oscillator oscillator is reviewed in given representation. In the third section we obtain equations for small and large components of a wavefunction and examine requirements imposed on the wave function. In the forth section energy spectrum of Dirac oscillator is obtained. In the fifth section wavefunctions of the problem are derived. Finally, the sixth section contains the conclusions.

\section{Dirac oscillator}

We consider stationary Dirac oscillator equation which can be written in the form:

$$
H \Psi=E \Psi, \quad H=\hat{\alpha}(\mathbf{P}-i m \omega \mathbf{X} \hat{\beta})+m \hat{\beta}
$$

where

$$
\hat{\alpha}=\left(\begin{array}{cc}
0 & \sigma \\
\sigma & 0
\end{array}\right) \hat{\beta}=\left(\begin{array}{cc}
I & 0 \\
0 & -I
\end{array}\right)
$$

and $\sigma_{i}, i=1,2,3$ are the Pauli matrices. We also put $\hbar=c=1$. It is supposed that position $X_{i}$ and momentum $P_{i}$ operators in the equation 1 are obeyed to deformed commutation relations which take form:

$$
\begin{aligned}
& {\left[X_{i}, P_{j}\right]=i\left(\delta_{i j}+\alpha X_{i} X_{j}+\beta P_{j} P_{i}+\sqrt{\alpha \beta}\left(P_{i} X_{j}+X_{j} P_{i}\right)\right),} \\
& {\left[X_{i}, X_{j}\right]=i \beta \varepsilon_{i j k} L_{k}, \quad\left[P_{i}, P_{j}\right]=i \alpha \varepsilon_{i j k} L_{k} .}
\end{aligned}
$$

Here $L_{k}$ are components of angular momentum operator and parameters $\alpha$ and $\beta$ are supposed to be positive. We also note, that there is summation over dummy indices. Components of angular momentum operator are defined as follows:

$$
J_{i j}=\varepsilon_{i j k} L_{k}=\frac{1}{2}\left(X_{i} P_{j}+P_{j} X_{i}-X_{j} P_{i}-P_{i} X_{j}\right)
$$

Components of angular momentum operator fulfil the ordinary commutation relations:

$$
\left[L_{i}, X_{j}\right]=i \varepsilon_{i j k} X_{k}, \quad\left[L_{i}, P_{j}\right]=i \varepsilon_{i j k} P_{k} .
$$

In the one-dimensional case the algebra (4) takes simpler form:

$$
[X, P]=i\left(1+\alpha X^{2}+\beta P^{2}+\sqrt{\alpha \beta}(P X+X P)\right)
$$


We note that similar one-dimensional deformed algebra was examined in the work [47] but in their case instead of factor $\sqrt{\alpha \beta}$ in the forth term in the right-hand side an independent parameter $\kappa$ was used. It is easy to show that the algebra (7) gives rise to uncertainty relation:

$$
\Delta X \Delta P \geqslant \frac{1}{2}\left|1+\gamma+\alpha(\Delta X)^{2}+\beta(\Delta P)^{2}+\sqrt{\alpha \beta}\langle\hat{X} \hat{P}+\hat{P} \hat{X}\rangle\right|
$$

where $\hat{X}=X-\langle X\rangle, \hat{P}=P-\langle P\rangle$ and $\gamma=(\sqrt{\alpha}\langle X\rangle+\sqrt{\beta}\langle P\rangle)^{2} \geqslant 0$. From the inequality $\mid\langle\hat{A} \hat{B}+$ $\hat{B} \hat{A}\rangle \mid \leqslant 2 \sqrt{\left\langle A^{2}\right\rangle\left\langle B^{2}\right\rangle}$ which is valid for any two operators $\hat{A}$ and $\hat{B}$ it follows that $|\langle\hat{X} \hat{P}+\hat{P} \hat{X}\rangle| \leqslant$ $2 \Delta X \Delta P$. Since parameters $\alpha$ and $\beta$ are positive, it leads to inequality $1+\gamma+\alpha(\Delta X)^{2}+\beta(\Delta P)^{2}>0$. Using these remarks we can rewrite the uncertainty relation (8) in the form:

$$
\Delta X \Delta P \geqslant \frac{1}{2}\left(1+\gamma+\alpha(\Delta X)^{2}+\beta(\Delta P)^{2}-2 \sqrt{\alpha \beta} \Delta X \Delta P\right) .
$$

The latter uncertainty relation brings minimal uncertainty in position as well as in momentum:

$$
\Delta X \geqslant(\Delta X)_{\min }=\sqrt{\frac{\beta(1+\gamma)}{1+2 \sqrt{\alpha \beta}}} ; \quad \Delta P \geqslant(\Delta P)_{\min }=\sqrt{\frac{\alpha(1+\gamma)}{1+2 \sqrt{\alpha \beta}}}
$$

It is important to emphasize that these minimal uncertainties do not appear if parameters $\alpha$ and $\beta$ are negative. Having done rescaling of uncertainties and parameters of deformation we can represent uncertainty relation in the well known form obtained by Kempf [40]:

$$
\Delta \bar{X} \Delta \bar{P} \geqslant \frac{1}{2}\left(1+\bar{\alpha}(\Delta \bar{X})^{2}+\bar{\beta}(\Delta \bar{P})^{2}\right)
$$

where

$$
\Delta \bar{X}=\sqrt{\frac{1+\sqrt{\alpha \beta}}{1+\gamma}} \Delta X, \quad \Delta \bar{P}=\sqrt{\frac{1+\sqrt{\alpha \beta}}{1+\gamma}} \Delta P, \quad \bar{\alpha}=\frac{\alpha}{1+\sqrt{\alpha \beta}}, \quad \bar{\beta}=\frac{\beta}{1+\sqrt{\alpha \beta}} .
$$

It is no doubt that "rescaled" uncertainty relation (11) leads to the same minimal uncertainties (10) as it should be.

In multidimensional case commutation relations (4) brings to uncertainty relation:

$$
\Delta X_{i} \Delta P_{j} \geqslant \frac{1}{2}\left|\delta_{i j}+\gamma_{i j}+\alpha\left\langle\hat{X}_{i} \hat{X}_{j}\right\rangle+\beta\left\langle\hat{P}_{j} \hat{P}_{j}\right\rangle+\sqrt{\alpha \beta}\left\langle\hat{P}_{i} \hat{X}_{j}+\hat{X}_{j} \hat{P}_{i}\right\rangle\right|
$$

where similarly as it was used in one dimensional case $\hat{X}_{i}=X_{i}-\left\langle X_{i}\right\rangle, \hat{P}_{i}=P_{i}-\left\langle P_{i}\right\rangle$ and $\gamma_{i j}=\alpha\left\langle X_{i}\right\rangle\left\langle X_{j}\right\rangle+\beta\left\langle P_{i}\right\rangle\left\langle P_{j}\right\rangle+2 \sqrt{\alpha \beta}\left\langle P_{i}\right\rangle\left\langle X_{j}\right\rangle$. It is easy to see that in case when $i=j$ the last relation reduces to $(9)$ and as a consequence the minimal uncertainties for position and momentum are the same as in the one-dimensional case (10)

To solve the Dirac equation (1) representation of operators $X_{i}, P_{j}$ that obeyed to commutation relations (4) should be defined. The algebra (4) does not have position or momentum representations because of noncommutativity of corresponding operators. To build up representation for position and momentum operators (4) it was proposed projective transformation [45] which introduce a relation between the commutation relations (4) and Snyder algebra [46]. As it was noted such a transformation is nonsymplectic. The position and momentum operators can represented as follows [45]:

$$
\begin{array}{r}
X_{i}=i \sqrt{1-\beta p^{2}} \frac{\partial}{\partial p_{i}}+\lambda \sqrt{\frac{\beta}{\alpha} \frac{p_{i}}{\sqrt{1-\beta p^{2}}}} \\
P_{i}=-i \sqrt{\frac{\alpha}{\beta}} \sqrt{1-\beta p^{2}} \frac{\partial}{\partial p_{i}}+(1-\lambda) \frac{p_{i}}{\sqrt{1-\beta p^{2}}} .
\end{array}
$$

Here $p^{2}=p_{k} p_{k}$ and parameter $\lambda$ is arbitrary real. Since $\alpha, \beta>0$ it leads to the restriction for the absolute value of square of variable $p: \beta p^{2}<1$. 
To provide hermicity of position and momentum operators scalar product should be defined with a weight function. It can be written in the form:

$$
\langle\psi \mid \varphi\rangle=\int \frac{d \mathbf{p}}{\sqrt{1-\beta p^{2}}} \psi^{*}(\mathbf{p}) \varphi(\mathbf{p})
$$

We note that according to abovementioned remark the domain of integration is bounded by the sphere: $p^{2} \leqslant 1 / \beta$. It is worth emphasizing that the weight function does not depend on the choice of parameter $\lambda$.

Components of the angular momentum operator defined by formula (5) are represented as follows:

$$
J_{i j}=\varepsilon_{i j k} L_{k}=i\left(p_{j} \frac{\partial}{\partial p_{i}}-p_{i} \frac{\partial}{\partial p_{j}}\right) .
$$

So the components of angular momentum operator take the same form as in momentum representation in ordinary quantum mechanics.

Wave function of the Dirac equation (1) can be written as a two-component spinor $\psi=\left(\begin{array}{l}\psi_{1} \\ \psi_{2}\end{array}\right)$ where functions $\psi_{1}$ and $\psi_{2}$ are called large and small component respectively. The Dirac equation (1) can be rewritten as a system of two coupled equations:

$$
\begin{aligned}
B^{+} \psi_{2} & =(E-m) \psi_{1}, \\
B^{-} \psi_{1} & =(E+m) \psi_{2} .
\end{aligned}
$$

where

$$
B^{ \pm}=(\boldsymbol{\sigma}, \mathbf{P}) \pm i m \omega(\boldsymbol{\sigma}, \mathbf{X})
$$

To get a factorized equation for the large component $\psi_{1}$ one should apply the operator $B^{+}(19)$ to the equation (18) and then in the right-hand sight of obtained equation the action of the operator on the component $\psi_{2}$ should be replaced by the right-hand side of the equation (17). As a result we arrive at:

$$
B^{+} B^{-} \psi_{1}=\left(E^{2}-m^{2}\right) \psi_{1} .
$$

Similarly for the small component $\psi_{2}$ we have:

$$
B^{-} B^{+} \psi_{2}=\left(E^{2}-m^{2}\right) \psi_{2} .
$$

The representation of position and momentum operators $X_{i}$ and $P_{j}$ allows one to get the explicit form for the operators $B^{ \pm}$:

$$
\begin{aligned}
& B^{+}=\left[-i\left(\sqrt{\frac{\alpha}{\beta}}-i m \omega\right) \sqrt{1-\beta P^{2}}\left(\frac{\partial}{\partial p}+\frac{(\boldsymbol{\sigma}, \mathbf{L})+2}{p}\right)+\left(1-\lambda+i m \omega \lambda \sqrt{\frac{\beta}{\alpha}}\right) \frac{p}{\sqrt{1-\beta p^{2}}}\right] \sigma_{p} \\
& B^{-}=\sigma_{p}\left[-i\left(\sqrt{\frac{\alpha}{\beta}}+i m \omega\right) \sqrt{1-\beta P^{2}}\left(\frac{\partial}{\partial p}-\frac{(\boldsymbol{\sigma}, \mathbf{L})}{p}\right)+\left(1-\lambda-i m \omega \lambda \sqrt{\frac{\beta}{\alpha}}\right) \frac{p}{\sqrt{1-\beta p^{2}}}\right]
\end{aligned}
$$

where $\sigma_{p}=(\boldsymbol{\sigma}, \mathbf{p}) / p$

The equations (17), (18) and as a consequence the operators $B^{ \pm}$would take simpler form if transformation of large and small functions is performed:

$$
\psi_{i}=\frac{1}{p} \varphi_{i}
$$

After that transformation equations (17) and (18) can be rewritten as follows:

$$
\begin{aligned}
\tilde{\omega} b^{+} \sigma_{p} \varphi_{2} & =(E-m) \varphi_{1}, \\
\tilde{\omega}^{*} \sigma_{p} b^{-} \varphi_{1} & =(E+m) \varphi_{2} .
\end{aligned}
$$

where $\tilde{\omega}=(m \omega+i \sqrt{\alpha / \beta})$ and $\tilde{\omega}^{*}$ is complex conjugate. Operators $b^{ \pm}$are obtained from relations (22), (23) and (24). They take form:

$$
b^{+}=-\sqrt{1-\beta p^{2}} \frac{\partial}{\partial p}-\frac{\sqrt{1-\beta p^{2}}}{p}((\boldsymbol{\sigma}, \mathbf{L})+1)+\eta \frac{p}{\sqrt{1-\beta p^{2}}}
$$




$$
b^{-}=\sqrt{1-\beta p^{2}} \frac{\partial}{\partial p}-\frac{\sqrt{1-\beta p^{2}}}{p}((\boldsymbol{\sigma}, \mathbf{L})+1)+\eta^{*} \frac{p}{\sqrt{1-\beta p^{2}}}
$$

here

$$
\eta=\frac{1-\lambda+i m \omega \lambda \sqrt{\frac{\beta}{\alpha}}}{m \omega+i \sqrt{\frac{\alpha}{\beta}}}=\frac{m \omega+i\left(m^{2} \omega^{2} \lambda \sqrt{\frac{\beta}{\alpha}}-\sqrt{\frac{\alpha}{\beta}}(1-\lambda)\right)}{m^{2} \omega^{2}+\frac{\alpha}{\beta}} .
$$

To simplify equations (26) and (26) one can introduce function:

$$
\tilde{\varphi}_{2}=\sigma_{p} \varphi_{2}
$$

As a result we arrive at

$$
\begin{aligned}
\tilde{\omega} b^{+} \tilde{\varphi}_{2} & =(E-m) \varphi_{1}, \\
\tilde{\omega}^{*} b^{-} \varphi_{1} & =(E+m) \tilde{\varphi}_{2} .
\end{aligned}
$$

\section{Superpartner hamiltonians and components of radial wave function}

Operators $b^{ \pm}(27)$ (28) introduced in the previous section commute with the total angular momentum $\mathbf{J}=\mathbf{L}+\mathbf{S}$ where $\mathbf{S}=\frac{1}{2} \boldsymbol{\sigma}$ as well as with $\mathbf{L}^{2}$ and $\mathbf{S}^{2}$, so the solutions $\varphi_{1}$ and $\tilde{\varphi}_{2}$ of equations (30) and (31) can be taken in the form representing the fact that they are eigenfunctions of operators $\mathbf{L}^{2}, \mathbf{S}^{2}, \mathbf{J}^{2}$ and $J_{z}$ with corresponding eigenvalues $l(l+1), 3 / 4, j(j+1)$ and $m$ respectively.

$$
\begin{aligned}
& \varphi_{1}=\varphi_{1}(p, s, j, m)=R_{1 ; s, j}(p) \mathcal{Y}_{s, j, m}(\theta, \varphi, \xi) \\
& \varphi_{2}=\varphi_{2}(p, s, j, m)=R_{2 ; s, j}(p) \mathcal{Y}_{s, j, m}(\theta, \varphi, \xi)
\end{aligned}
$$

where

$$
\mathcal{Y}_{s, j, m}(\theta, \varphi, \xi)=\sum_{\sigma, \mu}\left\langle j-s \mu, \frac{1}{2} \sigma \mid j m\right\rangle Y_{j-s, m}(\theta, \varphi) \chi_{\sigma}(\xi)
$$

is a spin spherical harmonic [48] and $R_{1 ; s, j}(p)$ and $R_{2 ; s, j}(p)$ are radial wavefunctions. It should be noted that $\chi_{\sigma}(\xi)$ denotes a spinor and $\sigma= \pm \frac{1}{2}$.

The main advantage of introduced function $\tilde{\varphi}_{2}$ is caused by the fact that it has the same spinangular part as the function $\varphi_{1}$. Whereas for the function $\varphi_{2}$ we have:

$$
\varphi_{2}=\sigma_{p} \tilde{\varphi}_{2}=\tilde{R}_{2 ; s, j}(p) \sigma_{p} \mathcal{Y}_{s, j, m}(\theta, \varphi, \xi)=-\tilde{R}_{2 ; s, j}(p) \mathcal{Y}_{-s, j, m}(\theta, \varphi, \xi)
$$

Last relation can be written as follows:

$$
\varphi_{2}=\varphi_{2 ;-s, j, m}(p, \theta, \varphi, \xi)=R_{2 ;-s, j}(p) \mathcal{Y}_{-s, j, m}(\theta, \varphi, \xi)
$$

and here $R_{2 ;-s, j}(p)=-\tilde{R}_{2 ; s, j}(p)$. We remark that wavefunctions $\phi_{1}$ and $\tilde{\phi}_{2}$ are characterized by the same value $l=j-s$.

To make equations (30) and (31) simpler we consider relation:

$$
((\boldsymbol{\sigma}, \mathbf{L})+1) \mathcal{Y}_{s, j, m}(\theta, \varphi, \xi)=\left(\mathbf{J}^{2}-\mathbf{L}^{2}-\mathbf{S}^{2}+1\right) \mathcal{Y}_{s, j, m}(\theta, \varphi, \xi)=s(2 j+1) \mathcal{Y}_{s, j, m}(\theta, \varphi, \xi) .
$$

Having used last equation one arrives at a system of coupled equations for radial wavefunctions:

$$
\begin{gathered}
\tilde{\omega} b_{p}^{+} \tilde{R}_{2}=(E-m) R_{1}, \\
\tilde{\omega}^{*} b_{p}^{-} R_{1}=(E+m) \tilde{R}_{2} .
\end{gathered}
$$

we use notation $b_{p}^{ \pm}$for the radial part of operators $b^{ \pm}$and they take form

$$
b_{p}^{+}=-\sqrt{1-\beta p^{2}} \frac{\partial}{\partial p}-\frac{k}{p} \sqrt{1-\beta p^{2}}+\frac{\eta p}{\sqrt{1-\beta p^{2}}} ;
$$




$$
b_{p}^{-}=\sqrt{1-\beta p^{2}} \frac{\partial}{\partial p}-\frac{k}{p} \sqrt{1-\beta p^{2}}+\frac{\eta^{*} p}{\sqrt{1-\beta p^{2}}}
$$

where $k=s(2 j+1)$.

In radial momentum space the scalar product (15) can be represented as follows:

$$
\left\langle R \mid R^{\prime}\right\rangle=\int_{0}^{1 / \sqrt{\beta}} \frac{d p}{\sqrt{1-\beta p^{2}}} R^{*}(p) R^{\prime}(p)
$$

It is easy to verify that with respect to the scalar product $(42)$ the operators $b_{p}^{+}(40)$ and $b_{p}^{-}(41)$ are mutually hermitian conjugates.

From equations (38) and (39) we obtain:

$$
\begin{aligned}
b_{p}^{+} b_{p}^{-} R_{1} & =\frac{1}{|\tilde{\omega}|^{2}}\left(E^{2}-m^{2}\right) R_{1} \\
b_{p}^{-} b_{p}^{+} \tilde{R}_{2} & =\frac{1}{|\tilde{\omega}|^{2}}\left(E^{2}-m^{2}\right) \tilde{R}_{2}
\end{aligned}
$$

The radial wavefunctions $R_{1}$ and $\tilde{R}_{2}$ can be treated as eigenfunctions of two superpartner hamiltonians $[49,50]$.

We consider bound state problem so normalizability condition should be imposed on the relativistic wavefunction $\psi=\left(\begin{array}{l}\psi_{1} \\ \psi_{2}\end{array}\right)$. It gives rise to the following relation:

$$
\int_{0}^{1 / \sqrt{\beta}} \frac{d p}{\sqrt{1-\beta p^{2}}}\left(\left|R_{1}\right|^{2}+\left|\tilde{R}_{2}\right|^{2}\right)=1 .
$$

In the presence of deformed commutation relations additional requirements are imposed on bound state wavefunctions. In case of uncertainty principle with minimal length it is demanded that any "physical" wavefunction belongs to the domain of operator $\mathbf{P}$ it means that meanvalue of square of momentum operator is finite. The deformed commutation relations (4) impose stricter requirements. To be acceptable a wavefunction should belong to the domains of operators $\mathbf{P}$ and $\mathbf{X}$. As a result it leads to finite meanvalues for square of both momentum and position.

Let us suppose that in the right-hand side of equations (43) and (44) we have eigenvalue $E^{2}=$ $\mathrm{m}^{2}$, so the corresponding wavefunctions are necessarily the solutions of equations:

$$
\begin{gathered}
b_{p}^{-} R_{1 ; 0}=0 \\
b_{p}^{+} \tilde{R}_{2 ; 0}=0
\end{gathered}
$$

Having integrated equation (46) we obtain:

$$
R_{1 ; 0}=C_{1 ; 0} p^{k}\left(1-\beta p^{2}\right)^{\frac{\tilde{\xi}}{2}+i \frac{\tilde{\zeta}}{2}}
$$

where $\tilde{\xi}=\frac{m \omega}{\alpha+\beta m^{2} \omega^{2}}$ and $\tilde{\zeta}=\frac{\sqrt{\alpha / \beta}(1-\lambda)-m^{2} \omega^{2} \lambda \sqrt{\beta / \alpha}}{\alpha+\beta m^{2} \omega^{2}}$ and $C_{1 ; 0}$ is the normalization constant.

The normalization condition (45) implies that integral from the square module of the function $R_{1 ; 0}$ must be finite:

$$
\int_{0}^{1 / \sqrt{\beta}} \frac{d p}{\sqrt{1-\beta p^{2}}}\left|C_{1 ; 0}\right|^{2} p^{2 k}\left(1-\beta p^{2}\right)^{\tilde{\xi}}=\left|C_{1 ; 0}\right|^{2} \int_{0}^{1 / \sqrt{\beta}} d p p^{2 k}\left(1-\beta p^{2}\right)^{\tilde{\xi}-\frac{1}{2}}<\infty
$$

For $p \rightarrow 0$ function $R_{1 ; 0}$ behaves as $p^{k}$ and boundary condition $R_{1 ; 0}=0$ leads to the restriction $k>0$ and this inequality is satisfied if $s=1 / 2$. When $p \rightarrow \frac{1}{\sqrt{\beta}}$ convergence of the integral (49) gives rise to the condition $\tilde{\xi}-\frac{1}{2}>-1$ or equivalently $\tilde{\xi}>-\frac{1}{2}$. But this inequality is always fulfilled because the parameter $\tilde{\xi}$ is defined as positive. So we conclude that wavefunction $R_{1 ; 0}$ is normalizable when $s=\frac{1}{2}$. 
As it has been already mentioned additional "physical" conditions should be imposed on the wave function $R_{1 ; 0} / p$. Meanvalues of square of momentum and position operators must be finite:

$$
\left\langle\frac{R_{1 ; 0}}{p}\left|\hat{P}^{2}\right| \frac{R_{1 ; 0}}{p}\right\rangle<\infty, \quad\left\langle\frac{R_{1 ; 0}}{p}\left|\hat{X}^{2}\right| \frac{R_{1 ; 0}}{p}\right\rangle<\infty .
$$

Meanvalue for square of momentum can be represented in the form:

$$
\left\langle\frac{R_{1 ; 0}}{p}\left|\hat{P}^{2}\right| \frac{R_{1 ; 0}}{p}\right\rangle=\int_{0}^{1 / \sqrt{\beta}} \frac{d p p^{2}}{\sqrt{1-\beta p^{2}}} \frac{R_{1 ; 0}^{*}}{p} \hat{P}_{p}^{2} \frac{R_{1 ; 0}}{p}<\infty
$$

where:

$$
\begin{array}{r}
\hat{P}_{p}^{2}=-\frac{\alpha}{\beta}\left(\left(1-\beta p^{2}\right)\left(\frac{1}{p^{2}} \frac{\partial}{\partial p} p^{2} \frac{\partial}{\partial p}-\frac{l(l+1)}{p^{2}}\right)-\beta p \frac{\partial}{\partial p}\right)- \\
2 i \sqrt{\frac{\alpha}{\beta}}(1-\lambda) p \frac{\partial}{\partial p}+(1-\lambda)\left((1-\lambda)-i \sqrt{\frac{\alpha}{\beta}}\right) \frac{p^{2}}{1-\beta p^{2}}-3 i \sqrt{\frac{\alpha}{\beta}}(1-\lambda)
\end{array}
$$

is the "radial" part of square of momentum operator. It should be noted that all remarks concerning meanvalue of square of momentum can be applied to the square of position operator because both of them have similar structure.

Taking into account the explicit form for operator $\hat{P}_{p}^{2}(52)$ and using requirement (51) one can obtain following condition for integral:

$$
\int_{0}^{1 / \sqrt{\beta}} d p \quad p^{2 k-2}\left(1-\beta p^{2}\right)^{\tilde{\xi}-\frac{3}{2}}<\infty
$$

It is easy to convince oneself that convergence of the latter integral in the vicinity of the point $p=0$ gives rise to the condition $k>0$. From the other side convergence of the integral (53) in the vicinity of the point $1 / \sqrt{\beta}$ will be provided if $\tilde{\xi}-\frac{3}{2}>-1$ from which we obtain restriction on the parameters of oscillator if it is supposed that parameters of deformation are held fixed:

$$
\frac{1}{\beta}(1-\sqrt{1-\alpha \beta})<m \omega<\frac{1}{\beta}(1+\sqrt{1-\alpha \beta})
$$

One can conclude that in order to obtain the eigenvalue $E^{2}=m^{2}$ in the equation (43) the condition $s=1 / 2$ should be required. We note that in case of two-parametric deformed algebra with minimal length eigenvalue $E^{2}=m^{2}$ exists also for positive projection of spin $s=1 / 2$ but an additional demand for values $j$ must be satified [35]. The mentioned requirement disappears in the limit case when one of those parameters corresponding to our parameter $\beta$ is kept.

Having integrated equation (47) we obtain:

$$
\tilde{R}_{2 ; 0}=C_{2 ; 0} p^{-k}\left(1-\beta p^{2}\right)^{-\frac{\tilde{\xi}}{2}+i \frac{\tilde{\xi}}{2}}
$$

Again the boundary conditions are imposed on it. For the first we require that $\tilde{R}_{2 ; 0} \rightarrow 0$ when $p \rightarrow 0$. The restriction $k<0$ or equivalently $s=-\frac{1}{2}$ follows immediately from the last requirement. From the other side one should demand $\tilde{R}_{2 ; 0} \rightarrow 0$ when $p \rightarrow \frac{1}{\sqrt{\beta}}$ but this requirement cannot be fulfilled because $-\frac{\tilde{\xi}}{2}<0$. As a result, the function $\tilde{R}_{2 ; 0}$ is not normalizable. To have physically acceptable function one should demand $\tilde{R}_{2 ; 0}=0$ and $R_{1 ; 0} \neq 0$. It is worth mentioning that the same requirement appears in case of two parametric deformed algebra with minimal length [35]. We also remark that the ground state wavefunction $\left(R_{1 ; 0} \neq 0, \tilde{R}_{2 ; 0}=0\right)$ is compatible with the positive eigenvalue $E=m$ whereas the negative one $E=-m$ will not be compatible with the system (38) and (39).

\section{Spectrum of Dirac oscillator}

In this section we will obtain energy spectrum for the Dirac oscillator. As it was shown in the previous section ground state with energy $E^{2}=m^{2}$ exists only for positive projection of spin $\left(s=\frac{1}{2}\right)$. In this section we will show that the ground state with energy $E^{2} \neq m^{2}$ can take place for positive $\left(s=\frac{1}{2}\right)$ as well as for negative $\left(s=-\frac{1}{2}\right)$ projection of spin. These two cases that correspond different ground state energy are considered separately. 


\subsection{Case of zero ground state energy}

As it has been already mentioned in the previous section that whenever $s=\frac{1}{2}$ and the condition (54) is fulfilled then equation (43) has acceptable wavefunction corresponding to the ground state energy $E^{2}-m^{2}=0$.

To solve eigenvalue problem (43) SUSY QM procedure is applied [49, 50]. Operator $h=b_{p}^{+} b_{p}^{-}$ is supposed to be the first member of the SUSY QM hierarchy

$$
h_{i}=b_{p}^{+}\left(k_{i}, \eta_{i}\right) b_{p}^{-}\left(k_{i}, \eta_{i}\right)+\sum_{j=0}^{i} \varepsilon_{j}, \quad i=0,1,2, \ldots
$$

Imposing shape invariance condition we obtain:

$$
b_{p}^{-}\left(k_{i}, \eta_{i}\right) b_{p}^{+}\left(k_{i}, \eta_{i}\right)=b_{p}^{+}\left(k_{i+1}, \eta_{i+1}\right) b_{p}^{-}\left(k_{i+1}, \eta_{i+1}\right)+\varepsilon_{i+1}
$$

In explicit form we write:

$$
\begin{aligned}
\eta_{i+1}-\eta_{i+1}^{*} & =\eta_{i}-\eta_{i}^{*} \\
k_{i+1}^{2}-k_{i+1} & =k_{i}^{2}+k_{i} \\
\frac{1}{\beta}\left|\eta_{i+1}\right|^{2}-\eta_{i+1}^{*} & =\frac{1}{\beta}\left|\eta_{i}\right|^{2}+\eta_{i} \\
-k_{i+1}^{2} \beta-k_{i+1}\left(\eta_{i+1}+\eta_{i+1}^{*}\right)-\frac{1}{\beta}\left|\eta_{i+1}\right|^{2} & +\varepsilon_{i+1}=-k_{i}^{2} \beta-k_{i}\left(\eta_{i}+\eta_{i}^{*}\right)-\frac{1}{\beta}\left|\eta_{i}\right|^{2}
\end{aligned}
$$

In the following we use notations: $\operatorname{Re} \eta_{i}=\xi_{i}$ and $\operatorname{Im} \eta_{i}=\zeta_{i}$ Having solved the first three equations we obtain:

$$
\zeta_{i}=\zeta, \quad \xi_{i}=\xi+\beta i, \quad k_{i}=k+i
$$

we note that $\xi=\beta \tilde{\xi}$ and $\zeta=\beta \tilde{\zeta}$. It is easy to show that for obtained values $\eta_{i}$ and $k_{i}$ the hierarchy hamiltonians $h_{i}$ have physically acceptable solutions $R_{1 ; 0}\left(k_{i}, \eta_{i}, p\right)$ corresponding to the energies $\sum_{j=0}^{i} \varepsilon_{j}$.

Having used the equation (61) we arrive at following equation for energy eigenvalues:

$$
E_{n}^{2}-m^{2}=\left(m^{2} \omega^{2}+\frac{\alpha}{\beta}\right) \sum_{j=0}^{n} \varepsilon_{j}=4 n\left(m^{2} \omega^{2}+\frac{\alpha}{\beta}\right)(\beta(n+k)+\xi)
$$

Since $k=s(2 j+1)$ and $\xi=\frac{m \omega}{m^{2} \omega^{2}+\alpha / \beta}$ the last relation can be rewritten in the form:

$$
E_{n}^{2}-m^{2}=4 n\left[m \omega+\left(m^{2} \omega^{2} \beta+\alpha\right)\left(n+j+\frac{1}{2}\right)\right]
$$

We note that in case $\alpha=0$ expression (64) is in agreement with corresponding relation obtained in the work [35] when one of their parameters of deformation is set to zero.

The principal quantum number $N=2 n+l=2 n+j-s$ can be introduced instead of $n$. Then the relation (64) can be represented as follows:

$$
E_{n}^{2}-m^{2}=2\left(N-j+\frac{1}{2}\right)\left[m \omega+\frac{1}{2}\left(m^{2} \omega^{2} \beta+\alpha\right)\left(N+j+\frac{3}{2}\right)\right] .
$$

\subsection{Nonzero ground state energy}

Now we suppose that in the right-hand side of the equations (43) and (44) we have $E^{2}-m^{2} \neq 0$. It will be shown that in this case the ground state exists for the following hamiltonian:

$h_{0}=b_{p}^{+}(k, \eta) b_{p}^{-}(k, \eta)=-\left(\sqrt{1-\beta p^{2}} \frac{\partial}{\partial p}\right)^{2}+\left(\eta-\eta^{*}\right) p \frac{\partial}{\partial p}+\frac{k^{2}-k}{p^{2}}+\frac{\frac{1}{\beta}|\eta|^{2}-\eta^{*}}{1-\beta p^{2}}-k\left(\eta+\eta^{*}\right)-k^{2} \beta-\frac{1}{\beta}|\eta|^{2}$

In order to obtain ground state energy one should re-factorize hamiltonian $h_{0}$. It can be represented as follows:

$$
h_{0}=b_{p}^{+}\left(k^{\prime}, \eta^{\prime}\right) b_{p}^{-}\left(k^{\prime}, \eta^{\prime}\right)+\varepsilon^{\prime},
$$


where $k^{\prime}$ and $\eta^{\prime}$ are new parameters in operators (40) and (41) and $\varepsilon^{\prime}$ defines the ground state energy. From equations (66) and (67) it follows:

$$
\begin{aligned}
\eta^{\prime}-\eta^{\prime *} & =\eta-\eta^{*} \\
k^{\prime 2}-k^{\prime} & =k^{2}-k \\
\frac{1}{\beta}\left|\eta^{\prime}\right|^{2}-\eta^{\prime} & =\frac{1}{\beta}|\eta|^{2}-\eta \\
-k^{\prime}\left(\eta^{\prime}+\eta^{\prime *}\right)-\beta k^{\prime 2}-\frac{1}{\beta}\left|\eta^{\prime}\right|^{2}+\varepsilon & =-k\left(\eta+\eta^{*}\right)-\beta k^{2}-\frac{1}{\beta}|\eta|^{2}
\end{aligned}
$$

Solving the equations (68)-(70) we arrive at the relations:

$$
\begin{aligned}
k_{1}^{\prime}=k, \quad k_{2}^{\prime}=1-k ; \\
\zeta^{\prime}=\zeta, \quad \xi_{1}^{\prime}=\xi, \quad \xi_{2}^{\prime}=\beta-\xi .
\end{aligned}
$$

Since conditions for parameters $k^{\prime}(72)$ and $\eta^{\prime}(73)$ are obtained independently then one can combine different $k^{\prime}$ and $\eta^{\prime}$ to investigate whether obtained wavefunctions will be physically acceptable.

For the first we consider the case $k^{\prime}=k$ and $\xi^{\prime}=\xi$. It follows immediately that $\varepsilon=0$. So the latter combination should be left out.

Then if we suppose that $k^{\prime}=k$ and $\xi^{\prime}=\beta-\xi$ equation (46) gives us corresponding wavefunction $R_{1 ; 0}=C_{1 ; 0} p^{k}\left(1-\beta p^{2}\right)^{\frac{\beta-\xi}{2 \beta}+i \frac{\zeta}{2 \beta}}$. The first requirements imposed on this function are boundary conditions. To provide the condition $R_{1 ; 0}=0$ at the boundaries one should demand that $k>0$ and $\beta-\xi>0$. As a consequence condition $k>0$ leads to requirement $s=\frac{1}{2}$ whereas the demand $\beta-\xi>0$ gives rise to $m \omega \in\left(0, \frac{1-\sqrt{1-4 \alpha \beta}}{2 \beta}\right)$ or $m \omega \in\left(\frac{1+\sqrt{1-4 \alpha \beta}}{2 \beta}, \infty\right)$. If $4 \alpha \beta>1$ the last condition can be satisfied for arbitrary $m \omega$. To make obtained wave function physically acceptable it must fulfil normalizability condition (49) and even stronger requirement (51). From the last requirement it follows that $-\frac{\xi}{\beta}+\frac{1}{2}>0$ and as a result it gives rise to the restrictions for the product $m \omega$ :

$$
m \omega \in\left(0, \frac{1-\sqrt{1-\alpha \beta}}{\beta}\right) \bigcup\left(\frac{1+\sqrt{1-\alpha \beta}}{\beta}, \infty\right) .
$$

One can see that obtained restrictions for the product $m \omega$ are opposite to (54). As a conclusion, if the relation (54) is fulfilled then the ground state has zero energy. If the condition (54) is broken the ground state with nonzero energy appears.

From relation (71) we obtain

$$
\varepsilon=(\beta-2 \xi)(1+2 k) .
$$

It is easy to verify that obtained ground state energy is positive.

To find other eigenvalues of the hamiltonian $h_{0}$ one should substitute $\xi^{\prime}$ instead of $\xi$ into (63) and take into account relation (75). After necessary transformations we arrive at:

$$
E_{n}^{2}-m^{2}=4(n+j+1)\left[-m \omega+\left(m^{2} \omega^{2} \beta+\alpha\right)\left(n+\frac{1}{2}\right)\right]
$$

Similarly as in previous case the obtained relation can be represented in terms of principal quantum number

$$
E_{n}^{2}-m^{2}=2\left(N+j+\frac{5}{2}\right)\left[-m \omega+\frac{1}{2}\left(m^{2} \omega^{2} \beta+\alpha\right)\left(N-j+\frac{3}{2}\right)\right] .
$$

From relations (72) and (73) it follows that ground states with nonzero energy other combinations of $k^{\prime}$ and $\eta^{\prime}$ are also possible. We consider combination $k^{\prime}=1-k$ and $\xi^{\prime}=\xi$. Then the ground state wavefunction takes form: $R_{1 ; 0}=C_{1 ; 0} p^{1-k}\left(1-\beta p^{2}\right)^{\frac{\xi}{2 \beta}+i \frac{\zeta}{2 \beta}}$. One of boundary conditions leads to restriction $k<0$ which can be satisfied if $s=-\frac{1}{2}$ and then $k^{\prime}=j+\frac{3}{2}$. Since $\xi>0$ the second boundary condition is satisfied immediately. It is easy to persuade oneself that obtained wavefunction is normalizable. Similarly as in the previous cases to make the obtained wavefunction physically acceptable we should impose condition (51) on it. Having used formula (71) we obtain following relation for the ground state energy:

$$
\varepsilon=(\beta+2 \xi)(1-2 k)
$$


One can see that ground state energy is also positive. Using the same procedure as in case with positive $k$ on can obtain energy spectrum:

$$
E_{n}^{2}-m^{2}=4(n+j+1)\left[m \omega+\left(m^{2} \omega^{2} \beta+\alpha\right)\left(n+\frac{1}{2}\right)\right]
$$

Having introduced the principal quantum number we rewrite the last relation in the form:

$$
E_{n}^{2}-m^{2}=2\left(N+j+\frac{3}{2}\right)\left[m \omega+\frac{1}{2}\left(m^{2} \omega^{2} \beta+\alpha\right)\left(N-j+\frac{1}{2}\right)\right] .
$$

In the end we can choose combination $k^{\prime}=1-k$ and $\xi^{\prime}=\beta-\xi$. This variant leads to the wave function $R_{1 ; 0}=C_{1 ; 0} p^{1-k}\left(1-\beta p^{2}\right)^{\frac{\beta-\xi}{2 \beta}+i \frac{\zeta}{2 \beta}}$. One of the boundary conditions gives rise to the demand $k<0$ or equivalently $k^{\prime}=j+\frac{3}{2}$. Another boundary condition leads to inequality $\beta-\xi>0$ but as we already know normalizability condition and boundness of the square of momentum operator should be satisfied and as a consequence all these demands lead to the condition (74).

For the ground state we obtain:

$$
\varepsilon=4(\beta(1-k)-\xi)
$$

It can be shown that the ground state energy (81) is positive if $4 \alpha \beta>1$. The same procedure leads us to the following expression for the spectrum:

$$
E_{n}^{2}-m^{2}=4(n+1)\left(-m \omega+\left(m^{2} \omega^{2} \beta+\alpha\right)\left(n+j+\frac{3}{2}\right)\right)
$$

Again we rewrite obtained formula replacing the quantum number $n$ by the principal quantum number $N$ :

$$
E_{n}^{2}-m^{2}=2\left(N-j+\frac{3}{2}\right)\left[-m \omega+\left(m^{2} \omega^{2} \beta+\alpha\right)\left(N+j+\frac{5}{2}\right)\right] .
$$

We note that this case does not have "classical" limit or in other words when parameters of deformation $\alpha, \beta \rightarrow 0$ obtained spectrum does not reduce to any solution of ordinary quantum mechanics [5]. Similar situation appears in the case of deformed algebra with minimal length [35].

\section{Radial momentum wavefunctions of Dirac oscillator}

In the previous section the ground state wavefunctions of hamiltonian $h=b_{p}^{+} b_{p}^{-}$have been derived. As it was shown only the large component of wavefunction can be obeyed to all imposed requirements. In this section we calculate the remaining large and small components of radial momentum wavefunction

\subsection{Zero ground state energy}

The large component of radial momentum wavefunction for excited states can be calculated with help of well-known SUSY QM and SI technique [49, 50]. As it is known the wave functions of the excited states are derived form the ground state wavefunction with help of recursive procedure which is based on the relation:

$$
R_{1 ; n}(p ; k, \xi)=\frac{1}{\sqrt{e_{n}-e_{0}}} b_{p}^{+}(k, \xi) R_{1 ; n-1}\left(p ; k_{1}, \xi_{1}\right) .
$$

Where we used notation $e_{i}=\left(E_{i}^{2}-m^{2}\right) /|\tilde{\omega}|^{2}$ for simplicity. According to the conditions (62) and (63) we should impose $e_{0}=0, k_{1}=k+1$ and $\xi_{1}=\xi+\beta$.

Having substituted the explicit form of operator $b_{p}^{+}$into the relation (84) we arrive at the equation:

$$
R_{1 ; n}(p ; k, \xi)=\frac{1}{\sqrt{e_{n}}}\left(-\sqrt{1-\beta p^{2}} \frac{\partial}{\partial p}-\frac{k}{p} \sqrt{1-\beta p^{2}}+\frac{(\xi+i \zeta) p}{\sqrt{1-\beta p^{2}}}\right) R_{1 ; n-1}(p ; k+1, \xi+\beta) .
$$


Given recursion procedure leads to consequence that the large component of radial wavefunction takes form:

$$
R_{1 ; n}(p ; k, \xi)=C_{1 ; n}(k, \xi) p^{b+\frac{1}{2}}\left(1-\beta p^{2}\right)^{\frac{a}{2}+\frac{1}{4}-i \frac{\zeta}{2 \beta}} P_{n}^{(a, b)}(z),
$$

where $C_{1 ; n}(k, \xi)$ and $P_{n}^{(a, b)}(z)$ are a normalization factor a Jacobi polynomial respectively. Here we also denoted:

$$
a=\frac{\xi}{\beta}-\frac{1}{2}, \quad b=k-\frac{1}{2}, \quad z=2 \beta p^{2}-1 \quad(-1<z<1) .
$$

It was argued in the previous section that the small component of the ground state radial wavefunction vanishes $\tilde{R}_{2 ; 0}(p ; k, \xi)=0$. For excited states small component can be found by using relation (39):

$$
\tilde{R}_{2 ; n}(p ; k, \xi)=\frac{\tilde{\omega}^{*}}{E_{n}+m} b_{p}^{-}(k, \xi) R_{1 ; n}(p ; k, \xi)
$$

Taking into account explicit expressions for the operator $b^{-}(k, \xi)(41)$ and wave function (86) one can rewrite the last relation in form:

$$
\begin{array}{r}
\tilde{R}_{2 ; n}(p ; k, \xi)=\frac{\tilde{\omega}^{*} C_{1 ; n}(k, \xi)}{E_{n}+m} \sqrt{1-\beta p^{2}}\left(\frac{\partial}{\partial p}-\frac{k}{p}+\frac{\eta^{*} p}{1-\beta p^{2}}\right) p^{b+\frac{1}{2}}\left(1-\beta p^{2}\right)^{\frac{a}{2}+\frac{1}{4}-i \frac{\zeta}{2 \beta}} P_{n}^{(a, b)}(z) \\
=\frac{\tilde{\omega}^{*} C_{1 ; n}}{E_{n}+m} \sqrt{1-\beta p^{2}}\left(\frac{\partial}{\partial p}-\frac{b+\frac{1}{2}}{p}+\frac{\left(\beta\left(a+\frac{1}{2}\right)-i \zeta\right) p}{1-\beta p^{2}}\right) p^{b+\frac{1}{2}}\left(1-\beta p^{2}\right)^{\frac{1}{2}\left(a+\frac{1}{2}\right)-i \frac{\zeta}{2 \beta}} P_{n}^{(a, b)}(z) \\
=\frac{2 \beta \tilde{\omega}^{*} C_{1 ; n}(k, \xi)(n+a+b+1)}{E_{n}+m} p^{b+\frac{3}{2}}\left(1-\beta p^{2}\right)^{\frac{1}{2}\left(a+\frac{3}{2}\right)-i \frac{\zeta}{2 \beta}} P_{n-1}^{(a+1, b+1)}(z) .
\end{array}
$$

It should be noted that a formula of differentiation of the Jacobi polynomials was used here $[51,52]$. In the previous section it was stated that wavefunction $\left(R_{1 ; 0}(p ; k, \xi) \neq 0, \tilde{R}_{2 ; 0}(p ; k, \xi)=0\right)$ is the physically acceptable solution of the system of equations (38) and (39) only for $E_{0}^{2}=m^{2}$. At the same time for excited states: $n=1,2, \ldots$ the solution of this system of equations is given by $\left(R_{1 ; n}(p ; k, \xi), \tilde{R}_{2 ; n}(p ; k, \xi)\right)$. It is necessary to verify whether these function are physically acceptable or not. It is easy to persuade oneself that the Jacobi polynomials in (86) and (89) do not spoil the convergence of integral (45) and also meanvalues for square of momentum and position operators would be finite similarly as it was given by the condition (51) for the ground state wavefunction. Finally, the normalization factor $C_{1 ; n}$ can be found from the normalization condition (45):

$$
C_{1 ; n}=\left(\beta^{b+1}(2 n+a+b+1) \frac{n ! \Gamma(n+a+b+1)}{\Gamma(n+a+1) \Gamma(n+b+1)} \frac{E_{n}+m}{E_{n}}\right)^{\frac{1}{2}}
$$

\subsection{Nonzero groundstate energy}

To find wavefunctions of excited states in remaining cases one should follow the approach used in the previous section. Parameters $k$ and $\xi$ in the iteration equation (84) should be replaced by $k^{\prime}$ and $\xi^{\prime}$ correspondingly. It worth noting that at the same time parameters $k$ and $\xi$ in the equation (88) remain unchanged. As a consequence we can state that equation (89) remains valid if parameters $a$ and $b$ are replaced by a new one.

In the case $k^{\prime}=k$ that corresponds $s=\frac{1}{2}$ and $\xi^{\prime}=\beta-\xi$ we obtain:

$$
R_{1 ; n}(p ; k, \xi)=C_{1 ; n} p^{b+\frac{1}{2}}\left(1-\beta p^{2}\right)^{\frac{1}{2}\left(a+\frac{1}{2}\right)-i \frac{\zeta}{2 \beta}} P_{n}^{(a, b)}(z)
$$

where $a=\frac{1}{2}-\frac{\xi}{\beta}$ and $b=k-\frac{1}{2}$.

The relation (88) gives rise to:

$$
\begin{array}{r}
\tilde{R}_{2 ; n}(p ; k, \xi)=\frac{\tilde{\omega}^{*} C_{1 ; n}}{E_{n}+m} \sqrt{1-\beta p^{2}}\left(\frac{\partial}{\partial p}-\frac{k}{p}+\frac{\eta^{*} p}{1-\beta p^{2}}\right) p^{b+\frac{1}{2}}\left(1-\beta p^{2}\right)^{\frac{1}{2}\left(a+\frac{1}{2}\right)-i \frac{\zeta}{2 \beta}} P_{n}^{(a, b)}(z) \\
=\frac{\tilde{\omega}^{*} C_{1 ; n}}{E_{n}+m} \sqrt{1-\beta p^{2}}\left(\frac{\partial}{\partial p}-\frac{b+\frac{1}{2}}{p}+\frac{\left(\beta\left(\frac{1}{2}-a\right)-i \zeta\right) p}{1-\beta p^{2}}\right) p^{b+\frac{1}{2}}\left(1-\beta p^{2}\right)^{\frac{1}{2}\left(a+\frac{1}{2}\right)-i \frac{\zeta}{2 \beta}} P_{n}^{(a, b)}(z) \\
=-\frac{2 \beta \tilde{\omega}^{*}(a+n) C_{1 ; n}}{E_{n}+m} p^{b+\frac{3}{2}}\left(1-\beta p^{2}\right)^{\frac{1}{2}\left(a-\frac{1}{2}\right)-i \frac{\zeta}{2 \beta}} P_{n}^{(a-1, b+1)}(z)
\end{array}
$$


In the case $k^{\prime}=1-k$ that corresponds $s=-\frac{1}{2}$ and $\xi^{\prime}=\xi$ we arrive at:

$$
R_{1 ; n}(p ; k, \xi)=C_{1 ; n} p^{b+\frac{1}{2}}\left(1-\beta p^{2}\right)^{\frac{1}{2}\left(a+\frac{1}{2}\right)-i \frac{\zeta}{2 \beta}} P_{n}^{(a, b)}(z)
$$

where $a=\frac{\xi}{\beta}-\frac{1}{2}$ and $b=\frac{1}{2}-k$.

Again the relation (88) leads to:

$$
\begin{array}{r}
\tilde{R}_{2 ; n}(p ; k, \xi)=\frac{\tilde{\omega}^{*} C_{1 ; n}}{E_{n}+m} \sqrt{1-\beta p^{2}}\left(\frac{\partial}{\partial p}-\frac{k}{p}+\frac{\eta^{*} p}{1-\beta p^{2}}\right) p^{b+\frac{1}{2}}\left(1-\beta p^{2}\right)^{\frac{1}{2}\left(a+\frac{1}{2}\right)-i \frac{\zeta}{2 \beta}} P_{n}^{(a, b)}(z) \\
=\frac{\tilde{\omega}^{*} C_{1 ; n}}{E_{n}+m} \sqrt{1-\beta p^{2}}\left(\frac{\partial}{\partial p}-\frac{\frac{1}{2}-b}{p}+\frac{\left(\beta\left(a+\frac{1}{2}\right)-i \zeta\right) p}{1-\beta p^{2}}\right) p^{b+\frac{1}{2}}\left(1-\beta p^{2}\right)^{\frac{1}{2}\left(a+\frac{1}{2}\right)-i \frac{\zeta}{2 \beta}} P_{n}^{(a, b)}(z) \\
=\frac{2 \beta \tilde{\omega}^{*}(b+n) C_{1 ; n}}{E_{n}+m} p^{b+\frac{3}{2}}\left(1-\beta p^{2}\right)^{\frac{1}{2}\left(a+\frac{3}{2}\right)-i \frac{\zeta}{2 \beta}} P_{n}^{(a+1, b-1)}(z)
\end{array}
$$

In the end we consider the case $k^{\prime}=1-k$ or equivalently as previously $s=-\frac{1}{2}$ and $\xi^{\prime}=\beta-\xi$. We arrive at:

$$
R_{1 ; n}(p ; k, \xi)=C_{1 ; n} p^{b+\frac{1}{2}}\left(1-\beta p^{2}\right)^{\frac{1}{2}\left(a+\frac{1}{2}\right)-i \frac{\zeta}{2 \beta}} P_{n}^{(a, b)}(z)
$$

where $a=\frac{1}{2}-\frac{\xi}{\beta}$ and $b=\frac{1}{2}-k$.

Having used relation (88) we obtain:

$$
\begin{array}{r}
\tilde{R}_{2 ; n}(p ; k, \xi)=\frac{\tilde{\omega}^{*} C_{1 ; n}}{E_{n}+m} \sqrt{1-\beta p^{2}}\left(\frac{\partial}{\partial p}-\frac{k}{p}+\frac{\eta^{*} p}{1-\beta p^{2}}\right) p^{b+\frac{1}{2}}\left(1-\beta p^{2}\right)^{\frac{1}{2}\left(a+\frac{1}{2}\right)-i \frac{\zeta}{2 \beta}} P_{n}^{(a, b)}(z) \\
=\frac{\tilde{\omega}^{*} C_{1 ; n}}{E_{n}+m} \sqrt{1-\beta p^{2}}\left(\frac{\partial}{\partial p}-\frac{\frac{1}{2}-b}{p}+\frac{\left(\beta\left(\frac{1}{2}-a\right)-i \zeta\right) p}{1-\beta p^{2}}\right) p^{b+\frac{1}{2}}\left(1-\beta p^{2}\right)^{\frac{1}{2}\left(a+\frac{1}{2}\right)-i \frac{\zeta}{2 \beta}} P_{n}^{(a, b)}(z) \\
=-\frac{2 \beta \tilde{\omega}^{*}(n+1) C_{1 ; n}}{E_{n}+m} p^{b+\frac{3}{2}}\left(1-\beta p^{2}\right)^{\frac{1}{2}\left(a-\frac{1}{2}\right)-i \frac{\zeta}{2 \beta}} P_{n+1}^{(a-1, b-1)}(z)
\end{array}
$$

It has been already noted that the last case does not have "classical" limit when the parameters of deformation $\alpha, \beta$ tend to zero. Similarly in the case of deformed algebra with minimal length [35] bounded states which do not have classical limit appear.

\section{Discussion}

In this work we considered the Dirac oscillator problem in deformed space given by the commutation relations (4). It was shown that deformed commutation relations (4) give rise to minimal uncertainty in position as well as in momentum. To find appropriate representation for position and momentum operators a specific nonsymplectic transformation was proposed [45]. It allows one to find some relation between given algebra (4) and well known Snyder algebra. Having used proposed representation it has been solved exactly the Dirac oscillator eigenvalue problem.

It has been shown that the Dirac oscillator in deformed space with commutation relations (4) has some common features with conventional case as well as in case of deformation with minimal length only. A dissymmetry under the exchange of $s=\frac{1}{2}$ with $s=-\frac{1}{2}$ that appeared in nondeformed case due to specific substitution $\mathbf{P} \rightarrow \mathbf{P}-i m \omega \mathbf{X} \hat{\beta}$ takes place in case of Snyder-de Sitter deformed algebra (4). The same situation happens in case of deformed algebra with minimal length [35]. If we consider system of equations (38) and (39) and make substitution $\omega \rightarrow-\omega$ the system can be transformed to equivalent one where $s$ is replaced by $-s$ and $E, R_{1}, \tilde{R}_{2}$ are changed into $-E,-\tilde{R}_{2}, R_{1}$ respectively. This transformation is valid in nondeformed case [5] and in the presence of deformed algebra with minimal length [35]. In nondeformed situation it is treated in connection with supersymmetry or, equivalently, with duality between particles and antiparticles [17]. Another similarity with previous cases lies in the absence of negative energy $E=-m$ ground states $[5,35]$.

It has been noted above the energy spectrum of the Dirac oscillator with deformed commutation relations (4) takes similar form as in case of deformed algebra with minimal length. [35]. In particular, the difference $E_{n}^{2}-m^{2}$ gets terms quadratic in $n$ instead of linear dependence in nondeformed instance. It should be noted that the relations for the energy spectrum would be in agreement with 
each other if the parameter $\alpha$ in our expressions is set to zero whereas in relations obtained in [35] the only parameter corresponding to our $\beta$ is kept. We also note that in case of deformed algebra with minimal length ground state with energy $E^{2}-m^{2}=0$ is allowed for small values $j$ only [35]. In contrast to it the Snyder-de Sitter algebra (4) does not make any restriction for parameter $j$ similarly as it was in ordinary quantum mechanics [5]. Ground states with nonvanishing energy $E^{2}-m^{2} \neq 0$ are allowed for both projections of spin: $s=\frac{1}{2}$ and $s=-\frac{1}{2}$. Here similarly to nondeformed situation no restriction on value of total angular momentum quantum number $j$ is imposed. It is worth stressing that in order to have physically acceptable wavefunctions parameters of oscillator should fulfil some conditions, namely product $m \omega$ can not take any value but it should satisfy such requirements as (54) or (74).

We also remark that although Dirac oscillator was introduced as a relativistic problem in our case it is not Lorentz covariant. This is caused by the fact that chosen algebra of operators (4) is not a relativistic one. The algebra (7) is obtained from the relativistic Snyder-de Sitter algebra [45, 42] and it seems that it easy to consider fully relativistic case but unfortunately some problems appear. The first one is that both time and energy will be represented by differential operators as we have here for position and momentum operators. The second problem is related to the behaviour of minimal uncertainties under Lorentz transformations. These questions need careful consideration and will examined elsewhere.

\section{References}

[1] D. Îto, K. Mori and E. Carriere, Nuovo Cimento A 51, 1119.

[2] P. A. Cook, Lett. Nuovo Cimento 1, 419 (1971).

[3] H. Ui, G. Takeda, Prog. Theor. Phys. 72, 266 (1984).

[4] A. B. Balantekin, Ann. Phys., NY 164, 277 (1985).

[5] M. Moshinsky, A. Szczepaniak, J. Phys. A: Math. Gen. 22 L817 (1989).

[6] M. Moreno, A. Zentella, J. Phys. A: Math. Gen. 22 L821 (1989).

[7] J. Benítez, R. P. Martínez y Romero, H. N. Núñez-Yépez and A. L. Salas-Brito, Phys. Rev. Lett. 641643 (1990); J. Benítez, R. P. Martínez y Romero, H. N. Núñez-Yépez and A. L. Salas-Brito, Phys. Rev. Lett. 6, 2085E (1990).

[8] C. Quesne, M. Moshinsky, J. Phys. A: Math. Gen. 23, 2263 (1990).

[9] O. L. de Lange, J. Phys. A: Math. Gen. 24, 667 (1991).

[10] J. Beckers, N. Debergh, Phys. Rev. D 42, 1255 (1990).

[11] R. P. Martinez y Romero, M. Moreno, A. Zentella, Phys. Rev. D 43, 2036 (1991).

[12] C. Quesne, Int. J. Mod. Phys. A 6, 1567 (1991).

[13] R. P. Martínez y Romero, A. L. Salas-Brito, J. Math. Phys. 33, 1831 (1992).

[14] R. Szmytkowski, M. Gruchowski, J. Phys. A: Math. Gen 344491 (2001).

[15] R. de Lima Rodrigues, Phys. Lett. A 372, 2587 (2008).

[16] S. Zarrinkamar, A. A. Rajabi, H. Hassanabadi, Ann. Phys. 325, 2522 (2010).

[17] M. Moshinsky, G. Loyola, Found. Phys. 23, 197 (1993).

[18] F. Domínguez-Adame, M. A. González, Europhys. Lett. 13, 193 (1990).

[19] A. Faessler, V. I. Kukulin, M. a. Shikhalev, Ann. Phys. 320, 71 (2005).

[20] J. Grineviciute, D. Halderson, Phys. Rev. C, 80, 044607 (2009).

[21] P. Rozmej, R. Arvieu, J. Phys. A 32, 5367 (1999).

[22] J. M. Torres, E. Sadurní, E. T. Seligman, AIP Conf. Proc., 1323, 301 (2010).

[23] A. Bermudez, M. A. Martin-Delgado, A. Luis. Phys. Rev. A, 77063815 (2008).

[24] A. Bermudez, M. A. Martin-Delgado, A. Luis. Phys. Rev. A, 77033832 (2008).

[25] E. Romera, Phys. Rev. A, 84, 052102 (2011). 
[26] Y. X. Wang, J. Cao, S. J. Xiong, Eur. Phys. J. B, 85, 237 (2012).

[27] S. Longhi, Opt. Lett. 35, 1302 (2010).

[28] J. A. Franco-Villafañe, E. Sadurni, S. Barkhofen et al., arXiv:1306.2204.

[29] D. J. Gross, P. F. Mende, Nucl. Phys. B 303, 407 (1988).

[30] M. Maggiore, Phys. Lett. B, 304, 65 (1993).

[31] E. Witten, Phys. Today, 49, 24 (1996).

[32] A. Kempf, G. Mangano and R. B. Mann, Phys. Rev. D 52 (1995) 1108.

[33] L. N. Chang, D. Minic, N. Okamura, T. Takeuchi, Phys. Rev. D, 65, 125027 (2002).

[34] Kh. Nouicer, J. Phys. A, 39, 5125 (2006).

[35] C. Quesne, V. M. Tkachuk, J. Phys. A 381747 (2005).

[36] T. V. Fityo, I. O. Vakarchuk, V. M. Tkachuk, J. Phys. A 39, 2143 (2006).

[37] C. Quesne, V. M. Tkachuk, J. Phys. A., 39, 10909 (2006).

[38] B. S. Kay, arXiv: hep-th/9802172; B.S. Kay, Class.Quant.Grav.15:L89-L98,1998; B. S. Kay, V. Abyaneh, arXiv: 0710.0992.

[39] B. Bagchi, A. Banerjee, C. Quesne, V. M. Tkachuk, J. Phys. A, 38, 2929 (2005).

[40] A. Kempf, J. Math. Phys., 35, 4483 (1994).

[41] C. Bambi, F. R. Urban, Class. Quant. Grav., 25, 095006 (2008).

[42] J. Kowalski-Glikman, L. Smolin, Phys. Rev. D, 70, 065020 (2004).

[43] C. Quesne, V. M. Tkachuk, J. Phys. A, 3610373 (2003).

[44] C. Quesne, V. M. Tkachuk, J. Phys. A, 37, 10095 (2004).

[45] S. Mignemi, arXiv:1110.0201.

[46] H. S. Snyder, Phys. Rev. 71, 38 (1947).

[47] C. Quesne, V. M. Tkachuk, SIGMA, 3, 016 (2007).

[48] A. R. Edmonds, Angular momentum in Quantum Mechanics, Princeton Univ. Press, Princeton (1957)

[49] F. Cooper, A. Khare, U. Sukhtame, Phys. Rept., 251, 267 (1995).

[50] G. Junker, Supersymmetric Methods in Quantum and Statistical Physics, Berlin, Springer (1996).

[51] A. Erdélyi, W. Magnus, F. Oberhettinger and F. G. Tricomi, Higher Transcendental Functions, vol.2, New York, McGraw-Hill (1953).

[52] Handbook of Mathematical Functions, ed. M. Abramowitz and I. Stegun, New York (1965). 